\title{
Electrocardiograph Voltage in Fit Young Men
}

\author{
Major Neil Ineson, \\ MRCP, RAMC
}

Dr S Patel, MRCP

Cpl A P Cowin, RAMC

L/CpI D S Jenkins, RAMC

\section{L/Cpl B Finan,} RAMC

\section{Lt Col Peter Lynch, $M D, M R C P$, RAMC}

Cardiac Department, Queen Elizabeth Military Hospital, Woolwich, London SEI8

ABSTRACT: Several criteria exist for the diagnosis for left ventricular hypertrophy as shown by high voltage ong ํㅐㄹ electrocardiograph. This study of 200 healthy young recruits to the Royal Artillery with normal blood pressurepand normal left ventricular wall thickness as measured by electrocardiography shows that no matter which criteria are $\$$ sied the false positive rate is approximately $25 \%$. High voltage is a normal phenomenon in young men and its use as a prediefor of left ventricular hypertrophy is likely to be misleading in this age group.

\section{Introduction}

The most commonly used method of determining left ventricular hypertrophy (LVH) from electrocardiographic voltage is that of Sokolow and Lyon ${ }^{1}$ whereby $\mathrm{LVH}$ is said to be present if the sum of the $S$ wave voltage in lead $V_{1}$ plus the $R$ wave voltage in $V_{5}$ or $V_{6}$ (whichever is the larger) is greater than 35 millivolts. Murphy et $\mathrm{al}^{2}$ found a false positive rate of $2 \%$ by this method in a study of 48 cardiologically normal autopsies of mean age 54 years. $\mathrm{Grant}^{3}$ modified this original system by suggesting that the voltage of the deeper $S$ wave of $V_{1}$ or $V_{2}$ be added to that of the taller $R$ wave of $V_{5}$ or $V_{6}$. Grubschmidt and Sokolow ${ }^{4}$ recognised that in young people these criteria would have to be modified and suggested the upper limit of normal should be rọsced from 35 millivolts to 40 millivolts in the age group $2 \overrightarrow{0}-\overline{85}$ years. In 1964, Manning and Smiley ${ }^{5}$ studied young Canadian airmen and recommended that this figure $\mathrm{Be}$ increased to 53 millivolts. Romhilt and Estest introduced a points system which included parameters other than voltage, and this was later modified Murphy ${ }^{8}$ (Table 1).

In the army, easily controlled hypertension does nō result in medical downgrading unless there is eviden of end-organ pathology. The inference of LVH fro voltage criteria is therefore important in the management of young hypertensives in the army.

Table 1:

Modified Romhilt Estes System ${ }^{8}$

1. $\mathrm{SV}_{1}+\mathrm{RV}_{5}$ or $\mathrm{RV}_{6}>35 \mathrm{~mm}$

2. Left axis deviation $-30^{\circ}$

3. QRS duration $>0.09$ seconds

4. T wave inversion in $\mathrm{V}_{6}>1 \mathrm{~mm}$

5. Left atrial abnormality

6. Intrinsicoid deflection in $V_{5}$ or $V_{6}>0.05$ seconds 
No of subjects

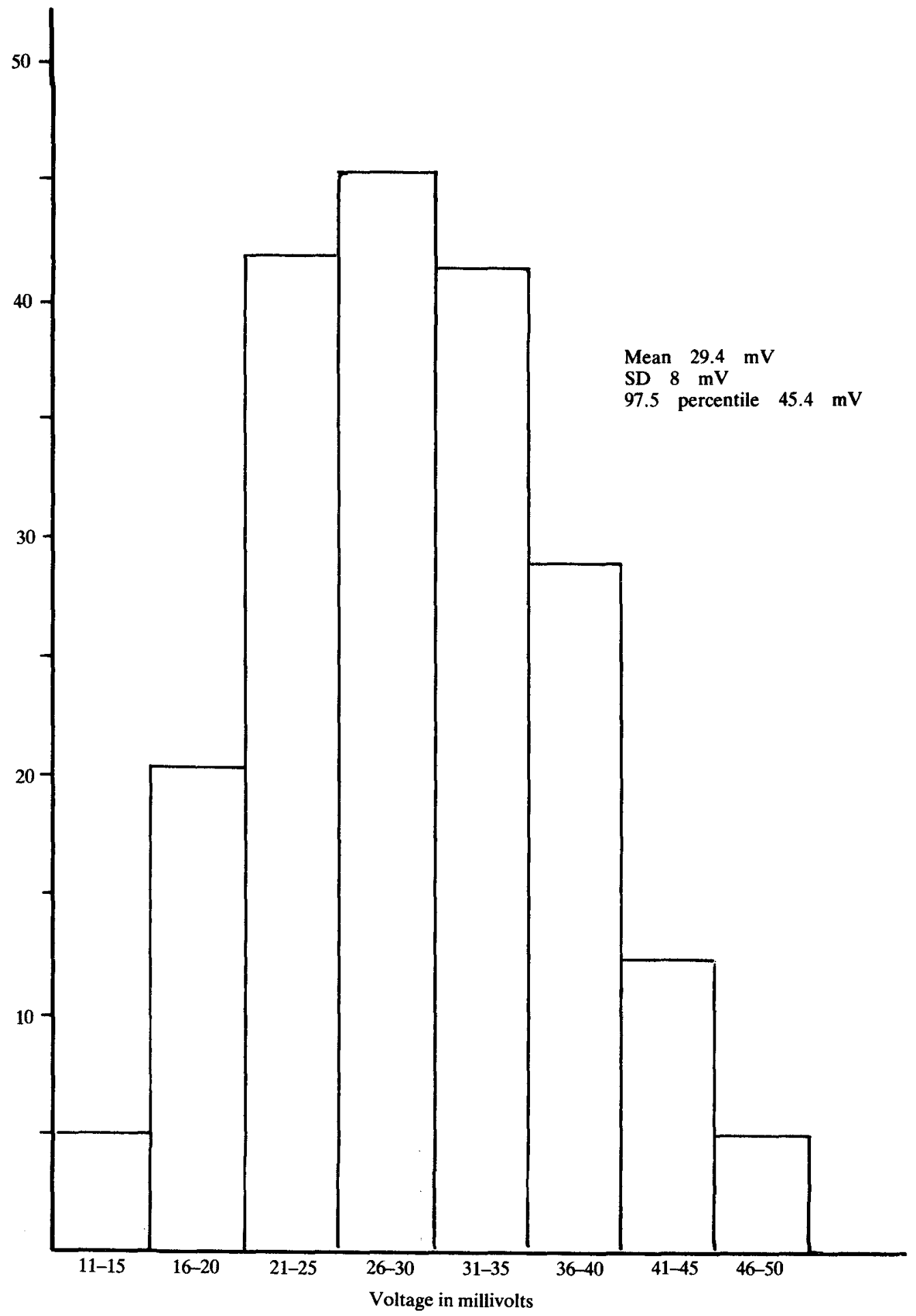

Figure 1: Sum of Voltages according to Sokolow $\left(\mathrm{SV}_{1}+\mathrm{RV}_{5}\right.$ or $\left.\mathrm{RV}_{6}\right)$ 


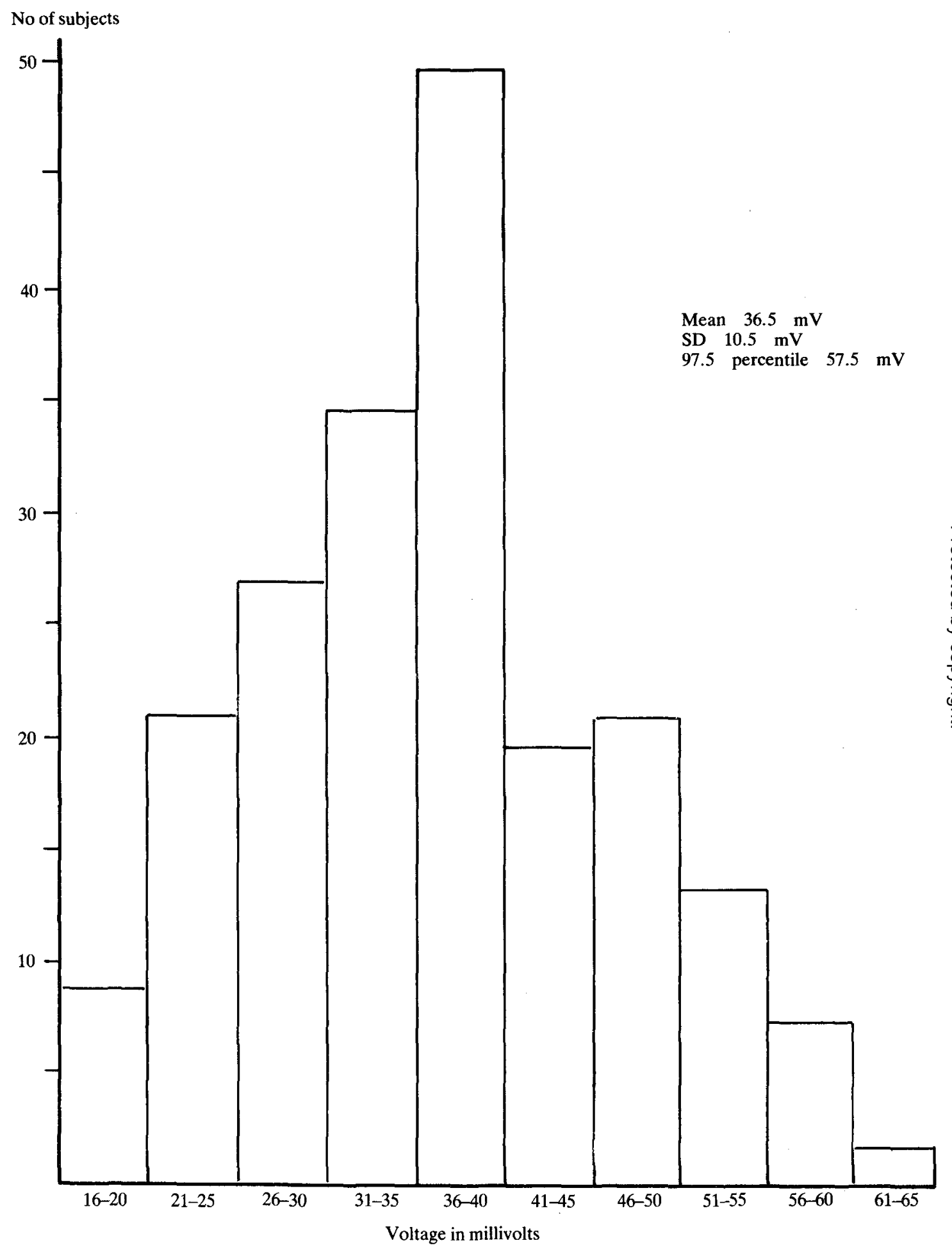

No of subjects

Figure 2: Sum of Voltages according to Grant $\left(\mathbf{S V}_{\mathbf{1}}\right.$ or $\mathbf{S V}_{\mathbf{2}}+\mathrm{RV}_{5}$ or $\left.\mathrm{RV}_{6}\right)$ 
Table 2:

Voltage criteria for left ventricular hypertrophy

\begin{tabular}{lcc}
\hline & no of patients & percentage \\
$\mathrm{SV}_{1}+\left(\mathrm{RV}_{5}\right.$ or $\left.\mathrm{RV}_{6}\right)>35 \mathrm{~mm}^{1}$ & 48 & 24 \\
$\left(\mathrm{SV}_{1}\right.$ or $\left.\mathrm{SV}_{2}\right)+\left(\mathrm{RV}_{5}\right.$ or $\left.\mathrm{RV}_{6}\right)>40 \mathrm{~mm}^{2}$ & 58 & 29 \\
fulfilling either criterion & 64 & 32 \\
fulfilling both criteria & 42 & 21 \\
\hline
\end{tabular}

1. Sokolow criteria

2. Grant criteria

\section{Subjects and Methods}

Two hundred consecutive recruits to the Royal Artillery of mean age 19 years (range 17-25 years) had clinical, electrocardiographic and echocardiographic examinations. All were normotensive, having a systolic blood pressure of less than 145 and a diastolic blood pressure of less than 90 millimeters of mercury when taken with a standard mercury sphygmomanometer. All fulfilled the criteria for enlistment as defined by the Joint Services Pulheems Classification System ${ }^{9}$. Twelve lead electrocardiographs were recorded, in semi-recumbent position, on Hewlett Packard Page Writer equipment (frequency response $0.05-100 \mathrm{Hertz}$ ) at 25 milimeters per second paper speed with standard calibration of 1 millimeter per millivolt. Both M-mode and two dimensional echocardiography were carried out using the Hewlett Packard 77020A Ultrasound Imaging System. Thickness of both the interventricular septum and the left ventricular posterior wall was measured, in diastole, on an axis through the chordae tendineae of both mitral cusps. In all cases septal thickness was less than 12 millimeters and posterior wall thickness less than 10 millimeters.

\section{Results}

The spectrum of QRS voltage by the Sokolow, and by the Grant method is shown in Figures 1 and 2 respectively. Twenty-four percent fulfilled the Sokolow and $29 \%$ the Grant criteria for left ventricular hypertrophy (Table 2), but none satisfied the criteria of the Romhilt Estes system.

\section{Discussion}

This study demonstrates that if a single criterion of LVH in the form of high QRS voltage is applied to a young normal population there will be a high incidence of false positive results. In Murphy's study ${ }^{2}$ the upper 97.5 percentile of 33 millivolts is significantly lower than the 45.4 millivolts found in this study. Even if the Sokolow modification for young people of 40 millivolts is applied, there is still a significant number of false positives. Our figures correspond closely to those of Manning and Smiley $^{4}$ who recorded a mean QRS voltage of 29.6 millivolts with the 97.5 percentile at 46.2 millivolts. The modified Romhilt Estes system ${ }^{8}$ showed no false positives but it is cumbersome and involves more parameters than voltage. The concept that electrocardiographic voltage by itself is an indicator of LVH should be abandoned in our young military population.

\section{REFERENCES}

1. SOKOLOW M and LYON T P. The ventricular complex in left ventricular hypertrophy as obtained by unipolar precordial and limb leads. Am Heart J 1949; 37: 161-86.

2. Murphy M L et al. Descriptive characteristics of the electrocardiogram from autopsied men free of cardiopulmonary disease - a basis for evaluating criteria for ventricular hypertrophy. Am J Cardiol 1983; 52: 1275-80.

3. Grant R P. Clinical electrocardiography. McGraw Hill, New York 1957, 77.

4. GrubschmidT H A and Sokolow M. The reliability of high voltage of the QRS complex as a diagnostic sign of left ventricular hypertrophy in adults. Am Heart $J$ 1957; 54: 689-94.

5. Manning G W and Smiley J R. QRS-voltage criteria for left ventricular hypertrophy in a normal male population. Circulation 1964; 29: 224-30.

6. Romhilt D W and Estes E H. A point-score system for the ECG diagnosis of left ventricular hypertrophy. Am Heart J 1968; 75: 752-8.

7. Romнilt D W et al. A critical appraisal of the electrocardiographic criteria for the diagnosis of left ventricular hyptertrophy. Circulation 1969; 40: 185-95.

8. MURPHY M L et al. Re-evaluation of electrocardiographic criteria for left, right and combined cardiac ventricular hypertrophy. Am J Cardiol 1984; 53: 1140-7.

9. Pulheems. A Joint Services system of medical classification 1976 para 0415. Joint Services Publication 346. 